\title{
UMA VIAGEM À PRODUÇÃo CIENTÍFICA EM QUALIDADE DE VIDA NO TRABALHO (QVT) NOS ANOS 2001 A 2005: estudo nos anais do ENANPAD
}

\author{
1- Jassio Pereira Medeiros \\ Universidade Federal Rural do Semi-Árido - UFERSA \\ jassiop@yahoo.com.br
}

\author{
2- José Arimatés Oliveira \\ Universidade Federal do Rio Grande do Norte - UFRN \\ arimates@ufrnet.br
}

Diego Maganhotto Coraiola - Editor

\section{RESUMO}

Este estudo se originou da dúvida relativa aos processos metodológicos e discursivos adotados nos trabalhos que investigam a QVT. Foram analisados 28 artigos publicados no ENANPAD, nos anos de 2001 a 2005. A investigação englobou, de acordo com a classificação de Bruyne, Herman e Schoutheete (1977), a análise das seguintes variáveis: (1) base epistemológica; (2) teórica; (3) morfológica. Em seguida buscou-se, conforme apontamentos de Gil (1991 e 1999), (4) traçar um perfil metodológico; para, depois, (5) levantar-se um padrão de referência; e a (6) demografia de autoria. Constatou-se que a maior parte dos trabalhos que vêm sendo desenvolvidos sobre QVT corresponde a um estágio pouco avançado do desenvolvimento de uma disciplina científica, contribuindo para a edificação de um quadro conceitual descritivo que tem, no máximo, um poder de generalização empírica.

Palavras-Chave

Qualidade de vida no trabalho; Estado da arte; Produção científica.

\section{ABSTRACT}

This study was originated from the relative doubt to the methodology and discursive processes adopted in the papers that investigate the QWL. Had been analyzed 28 articles published in the ENANPAD, in the years 2001 until 2005. The inquiry approached, in accordance with the classification of Bruyne, Herman and Schoutheete (1977), the analysis of the following variable: (1) epistemology base; (2) theoretical base; (3) morphologic base. After that, was searched as notes of Gil (1991 and 1999), (4) to trace a methodology profile; for, later, (5) to arise a reference standard; e (6) the demography of authorship. It was evidenced that most of the papers that come being developed on QWL corresponds to a little advanced situation of the development of one disciplines scientific, contributing for the construction of an widely descriptive conceptual picture.

Keywords

Quality of work life; State of the art; Scientific production. 


\section{O Mapa}

A produção acadêmica em Administração, no Brasil, duplicou na década de 1990. Isso levou a uma preocupação com a qualidade dessa produção, principalmente, em virtude do grande número de balanços retrospectivos, essencialmente críticos, feitos a partir de 1990 e mais intensamente após 1997 (TONELLI et al, 2003). Estudos com essa finalidade surgiram e se multiplicaram nas áreas de organizações, marketing, administração da informação, produção, administração pública e recursos humanos, no entanto, ainda não preencheram todas as lacunas existentes dentro desse campo de pesquisa.

Seguindo esta tendência, pode-se também indagar: De que forma está sendo conduzida a produção científica sobre Qualidade de Vida no Trabalho (QVT) no século XXI dentro dos anais do ENANPAD e o que se pode comentar da sua relevância? Este artigo é uma tentativa de responder, ainda que parcialmente estas questões, uma vez que se baseia na análise de 28 artigos publicados no ENANPAD - Encontro da Associação Nacional dos Programas de PósGraduação em Administração - de 2001 a 2005, versando sobre o tema QVT.

A temática da QVT pode ser expressa, no Brasil, a partir de consulta ao acervo disponibilizado nos sites das principais revistas nacionais de Administração, segundo a classificação "Qualis das áreas", divulgada pela CAPES; a saber: Revista de Administração de Empresas - RAE, Revista de Administração Contemporânea - RAC, Revista de Administração Pública-RAP, Revista de Administração da Universidade de São Paulo RAUSP, Revista Eletrônica de Administração READ e a Revista Organização e Sociedade O\&S.

Na época da consulta realizada durante o mês de março de 2006, a RAE disponibilizava em seu site, um acervo com números de janeiro de 1961 a dezembro de 2005. A RAC apresentava números de setembro de 1997 a dezembro de 2005; a RAP, de janeiro de 2000 a agosto de 2005; a RAUSP, de janeiro de 1977 a dezembro de 2005; a READ, de setembro de 1995 a agosto de 2005 e a O\&S de maio de 1999 até dezembro de 2004.

O que se pôde constatar foi que os estudiosos brasileiros da QVT não costumam publicar seus artigos em periódicos, visto que dentre todos os números de publicações pesquisados junto aos periódicos, apenas 13 artigos foram encontrados, em quatro dessas revistas (RAP, RAE, RAC e RAUSP). A RAUSP, revista que mais publicou artigos sobre o tema, apresentou seis, em 28 anos de acervo. A RAE, apenas três em 44 anos de acervo; a RAC tinha dois, em oito anos de números disponibilizados em seu site; e a RAP também dois, em cinco anos. A O\&S e a READ, provavelmente por serem periódicos com menor tempo de publicações, não apresentaram nenhuma publicação referente ao tema.

A avaliação dos trabalhos é guiada por quatro critérios básicos: as bases epistemológica, teórica e morfológica e o levantamento do perfil metodológico dos artigos. Os três primeiros critérios são medidos de acordo com Bruyne, Herman e Schoutheete (1977) e o último, de acordo com Gil (1991, 1999). Assim, pretende-se auxiliar o investigador que pesquisa sobre o construto da qualidade de vida no trabalho e, ao mesmo tempo, contribuir para a diminuição dos estudos que não agregam novos conhecimentos para o tema.

O presente estudo é parte integrante de dissertação de mestrado defendida em 2007 e se originou da dúvida relativa aos procedimentos metodológicos e processos discursivos adotados nos trabalhos que investigam a QVT. Baseado em uma análise documental apresenta sua relevância na constatação de que existem lacunas na área de recursos humanos referentes a balanços críticos relacionados à produção científica sobre temas específicos como é a qualidade de vida no trabalho. Assim, é que se pretende enriquecer e ampliar o debate sobre o rumo que vem sendo tomado pelas pesquisas na área.

Após a apresentação deste 'mapa' de viagem, são retratados, na seqüência, o 'marco' do estudo fundado na evolução das pesquisas sobre a produção científica em administração - e o percurso seguido pela pesquisa. Quanto ao que foi encontrado, observou-se a predominância de artigos hipotético-dedutivos, sob um quadro de análise tipológico, referenciado pelo positivismo, com abordagem qualitativo-quantitativa, descritivos, predominando a técnica do levantamento de dados.

Como 'lugar onde se pôde chegar', observou-se que há críticas colocadas por trabalhos anteriores realizados na área de Recursos Humanos e comprovadas por este estudo. Essas se referem, principalmente, ao caráter empirista analítico e ao uso de um quadro conceitual amplamente descritivo; no entanto, há algumas evoluções no que tange ao aumento do número de pesquisas quali-quantitativas, e uma maior dispersão dos autores que estão publicando sobre o tema, talvez por representarem instituições diferentes. Por fim, ainda são questionados alguns preconceitos vigentes em relação ao estudo de caso. 


\section{O Marco (Teórico Conceitual)}

Tem crescido consideravelmente nos últimos anos, diversos estudos acerca da produção científica em administração no Brasil. Essa literatura vem abrangendo múltiplas dimensões e variáveis de análise em quase todas as áreas da administração. O trabalho pioneiro de Machado, Cunha e Amboni (1988) parece ser aquele que despertou a academia de Administração para a necessidade de uma ampla avaliação de sua produção. Os autores fizeram uma avaliação dos artigos da área de Administração Pública, a partir de artigos apresentados à ANPAD, no período de 1983 a 1986, concluindo pela produção incipiente, ênfase em problemáticas nacionais e uma forte orientação de cunho predominantemente teórico.

Parte dos trabalhos que se seguiram analisaram uma área temática, como na área de Marketing (VIEIRA, 1998, 1999, 2000, 2003; PERIN et al., 2000; FROEMMING et al, 2000; FROEMMING et al 2000b; PROENÇA; LOPES; MEUCCI, 2005); de Administração da Informação (HOPPEN et al., 1998; LUDMER et al, 2002; RAMOS; SILVA; SALES, 2004; LUNARDI; RIOS; MAÇADA, 2005); de Produção (BIGNETTI; PAIVA, 2002; ARKADER, 2003); de Administração Pública (MACHADO; CUNHA; AMBONI, 1989; KEINERT, 1994, 2000; GONÇALVES; GONÇALVES; AUGUSTO, 2003); de Finanças (LEAL; OLIVEIRA; SOLURI, 2003; THEÓPHILO; IUDÍCIUS, 2005; CAMARGOS; COUTINHO; AMARAL, 2005); Estratégia Organizacional (BIGNNETI e PAIVA, 2002; RODRIGUES FILHO, 2004; GOSLING; GONÇALVES, 2004; COSTA, 2005; PRADO JR.; LOEBEL, 2005; PEGINO, 2005); Organizações (MACHADO; CUNHA; AMBONI, 1990; HEMAIS; VERGARA, 2001; PAIVA JR.; CORDEIRO, 2002); Recursos Humanos (SIQUEIRA, 1988; BASTOS, 1989; TONELLI et al, 2003); além da Administração de uma maneira geral (MARTINS, 1993; MARTINS, 1996; BERTERO; CALDAS; WOOD JR., 1999; MATTOS, 2002; KIRSHBAUM; PORTO; FERREIRA, 2004).

É possível perceber que, em 2005, há uma tendência no ENANPAD à realização de metaestudos que analisam, de um lado, os próprios anais do Encontro e de outro, periódicos nacionais e internacionais. Esses estudos reportam-se a áreas temáticas de uma forma geral (como citado no parágrafo anterior) ou a temas específicos dentro das diversas áreas temáticas adotadas pela associação, como Responsabilidade Social (PASSADOR; CANOPF; PASSADOR, 2005) e Ética (CALDAS; ONKEN; TINOCO, 2005). Seja para uma área, para várias ou para um tema específico, ao mesmo tempo, estes estudos têm focado aspectos específicos, tais como o mapeamento de perfis epistemológicos, metodológicos, tipo e origem de referência bibliográfica, bases filosóficas das publicações entre outros.

Nesse sentido, Roesch, Antunes e Silva (1997) pesquisaram tendências de estudos em recursos humanos, analisando dissertações de mestrado nas décadas de 1980 e 1990. Os autores mostraram que existe uma predominância de estudos quantitativos em RH. Outro tipo de estudo que se tornou popular é o que avalia o tipo, origem e padrão de citações da produção científica. Caldas, Tinoco e Chu (2003) exemplificam esse tipo de pesquisa analisando a produção acadêmica em Recursos Humanos no Brasil, na década de 1990. Os resultados desse estudo mostraram uma área com alto índice de auto-citação e endogenia, e ainda uma elevada incidência de citação de jornais, revistas tipo magazine e autores estrangeiros e não acadêmicos.

Apesar de novo, como campo científico no Brasil, a pesquisa em administração de empresas tem crescido quantitativamente nos últimos anos. Entretanto, parece que a qualidade não tem acompanhado a quantidade, o que torna a produção periférica, epistemologicamente falha, metodologicamente deficiente, sem originalidade e prática, em grande escala, baseada em um mimetismo mal informado (BERTERO; CALDAS; WOOD JR., 1999).

A produção acadêmica na área de Recursos Humanos, então, até a década de 1980 era escassa e pontual, sendo quase toda a literatura proveniente de outros países e, portanto, não representativa dos problemas locais. A partir dos anos 1980, devido, em parte, à maior visibilidade que a área de Recursos Humanos ganha no contexto internacional e à criação da área no ENANPAD, em 1982, percebe-se um crescente interesse da academia pela área e os temas a ela relacionados.

Com relação a um crescimento quantitativo significativo da difusão de balanços críticos, até 2003 a área de recursos humanos ainda não tinha feito um levantamento amplo de sua produção científica, embora dois estudos (SIQUEIRA, 1988; ROESCH; ANTUNES; SILVA, 1997) tenham procurado iniciar e incentivar esse tipo de levantamento. Nesse ano Tonelli e outros apresentam um perfil da produção acadêmica na área, a partir da análise de todos os artigos publicados em periódicos científicos nacionais e anais do ENANPAD, na década de 1990. 
Esse estudo constatou que, embora a produção da área tenha aumentado significativamente em volume, o perfil acadêmico de recursos humanos no Brasil é preocupante, de maneira que seu escopo temático é contestado pelo recente crescimento e autonomia do campo de comportamento organizacional; sua base epistemológica é eminentemente funcionalista; a base metodológica é frágil, predominando estudos de caso tipicamente ilustrativos de teoria consolidada, onde a diversidade de origem é baixa: mais de $65 \%$ da produção vem de apenas sete programas de pós-graduação.

Dentro desse contexto, Tonelli et al (2004) se refere ao tema QVT como um dos principais figurantes entre os trabalhos, agora publicados nas áreas de Comportamento Organizacional e Gestão de Pessoas (a partir de 2001 a área de Recursos Humanos do ENANPAD foi dividida em duas: Gestão de Pessoas e Comportamento Organizacional). Ainda segundo os autores, tratase de um tema bastante abrangente, onde podem ser encontrados trabalhos sobre diversos subtemas como os programas de QVT sendo utilizados, os facilitadores e dificultadores para a implantação dos programas, a relação com a satisfação e o comprometimento, as múltiplas relações com a saúde dos trabalhadores, além das questões do estresse e do equilíbrio entre vida pessoal e profissional.

Apesar de fundamentais para compreender 0 campo de estudos sobre recursos humanos, esses trabalhos não são, nem pretendiam ser suficientes para se traçar um panorama abrangente a respeito da pesquisa no Brasil. As recomendações propostas por Caldas, Tonelli e Lacombe (2002) indicam que parece ser não só oportuno como essencial que a lacuna deixada pelos estudos até então desenvolvidos seja preenchida e um amplo debate possa ser estabelecido. Assim, pretende-se aqui aprofundar esses "meta-estudos", agora enfocando tema específico da área do Comportamento Organizacional, a Qualidade de Vida no Trabalho.

\section{O Percurso}

O presente estudo analisou todos os artigos aceitos sobre o tema 'Qualidade de Vida no Trabalho' (QVT) nos ENANPADs do século XXI, ou seja, durante os cinco últimos anos (2001, 2002, 2003, 2004 e 2005). A investigação englobou, de acordo com a classificação de Bruyne, Herman e Schoutheete (1977), a análise das seguintes variáveis: (1) base epistemológica. (2) base teórica; (3) base morfológica. Em seguida buscou-se, conforme apontamentos de Gil (1991, 1999), (4) traçar um perfil metodológico; para, depois, (5) levantar-se um padrão de referência; e a (6) demografia de autoria. Nesta seção, são relatados os procedimentos de coleta, bem como os critérios de categorização usados no estudo.

A base de dados partiu dos artigos digitalizados nos Anais do Encontro da Associação Nacional dos Programas de Pós-graduação em Administração (ENANPAD) dos anos de 2001 a 2005, conforme quadro a seguir. Cada um dos 28 artigos foi lido e as variáveis categorizadas (codificadas) pelo pesquisador. Para evitar desvios de classificação em função da óbvia subjetividade envolvida no trabalho de codificação, o trabalho de coleta e inserção de informações no banco de dados teve uma re-conferência para todas as variáveis. Buscase, assim, desenvolver procedimentos metodológicos convenientes, mesmo sabendo que nenhum método garante o acesso privilegiado à verdade.

\begin{tabular}{|c|c|}
\hline ANO & ARTIGO \\
\hline \multirow[t]{3}{*}{2001} & $\begin{array}{l}\text { HONORIO, L.; MARQUES, A.; MELO, M. Qualidade de vida no trabalho em uma micro empresa do comércio varejista. In: } \\
\text { Encontro da Associação Nacional dos Programas de Pós-Graduação em Administração, 25., 2001, Campinas. Anais... Rio } \\
\text { de Janeiro: ANPAD, } 2001 .\end{array}$ \\
\hline & $\begin{array}{l}\text { MORAES, L F R; et al. Estresse e qualidade de vida no trabalho na polícia militar do estado de Minas Gerais. In: Encontro da } \\
\text { Associação Nacional dos Programas de Pós-Graduação em Administração, 25., 2001, Campinas. Anais... Rio de Janeiro: } \\
\text { ANPAD, } 2001 .\end{array}$ \\
\hline & $\begin{array}{l}\text { OLIVEIRA, R.; MORAES, L. Qualidade de vida no trabalho: uma análise no contexto de trabalho dos detetives da Polícia } \\
\text { Civil Metropolitana de Belo Horizonte. In: Encontro da Associação Nacional dos Programas de Pós-Graduação em } \\
\text { Administração, 25., 2001, Campinas. Anais... Rio de Janeiro: ANPAD, 2001. }\end{array}$ \\
\hline \multirow[t]{2}{*}{2002} & $\begin{array}{l}\text { BRIGHENTI, G.; SILVA, A.; FERNANDES, C. Qualidade de vida no trabalho: um estudo dos fatores e dimensões presentes } \\
\text { e percebidas na central de relacionamento com os clientes da TIM celular de Santa Catarina. In: Encontro da Associação } \\
\text { Nacional dos Programas de Pós-Graduação em Administração, 26., 2002, Salvador. Anais... Rio de Janeiro: ANPAD, } 2002 .\end{array}$ \\
\hline & $\begin{array}{l}\text { GUEIROS, M.; OLIVEIRA, L. Qualidade de vida no trabalho: um estudo no setor de hotelaria na Região Metropolitana do } \\
\text { Recife. In: Encontro da Associação Nacional dos Programas de Pós-Graduação em Administração, 26., 2002, Salvador. } \\
\text { Anais... Rio de Janeiro: ANPAD, } 2002 .\end{array}$ \\
\hline
\end{tabular}




\begin{tabular}{|c|c|}
\hline & $\begin{array}{l}\text { KILIMNIK, Z.; CASTILHO, I. Trajetórias e transições de carreira: um estudo longitudinal sobre a qualidade de vida de } \\
\text { profissionais assalariados de recursos humanos que experimentaram a passagem para o trabalho autônomo. In: Encontro da } \\
\text { Associação Nacional dos Programas de Pós-Graduação em Administração, 26., 2002, Salvador. Anais... Rio de Janeiro: } \\
\text { ANPAD, 2002. }\end{array}$ \\
\hline & $\begin{array}{l}\text { OLIVEIRA, N. Mudanças Organizacionais e Qualidade de vida no trabalho: um estudo comparativo-temporal em unidades do } \\
\text { Banco do Brasil S.A. In: Encontro da Associação Nacional dos Programas de Pós-Graduação em Administração, 26., 2002, } \\
\text { Salvador. Anais... Rio de Janeiro: ANPAD, } 2002 .\end{array}$ \\
\hline & $\begin{array}{l}\text { OLIVEIRA, P.; LIMONGI FRANÇA, A.; MURITIBA, S. Avaliação de resultados em RH: fontes de evidência da percepção e } \\
\text { das práticas dos administradores no caso dos programas de qualidade de vida no trabalho. In: Encontro da Associação } \\
\text { Nacional dos Programas de Pós-Graduação em Administração, 26., 2002, Salvador. Anais... Rio de Janeiro: ANPAD, } 2002 .\end{array}$ \\
\hline & $\begin{array}{l}\text { PAIVA, K.; et al. Situação de trabalho, qualidade de vida e estresse no ambiente acadêmico: comparando professores de } \\
\text { instituições pública, privada e confessional. In: Encontro da Associação Nacional dos Programas de Pós-Graduação em } \\
\text { Administração, 26., 2002, Salvador. Anais... Rio de Janeiro: ANPAD, } 2002 .\end{array}$ \\
\hline & $\begin{array}{l}\text { PICCININI, V.; OLIVEIRA S. Flexibilização, qualidade de vida e empregabilidade: o caso das cooperativas de trabalho de } \\
\text { Porto Alegre. In: Encontro da Associação Nacional dos Programas de Pós-Graduação em Administração, 26., 2002, } \\
\text { Salvador. Anais... Rio de Janeiro: ANPAD, 2002. }\end{array}$ \\
\hline & $\begin{array}{l}\text { SILVA, L.; ANTUNES, E. Influência das relações flexíveis de trabalho na qualidade de vida do trabalhador: a visão de seus } \\
\text { atores sociais. In: Encontro da Associação Nacional dos Programas de Pós-Graduação em Administração, 26., 2002, } \\
\text { Salvador. Anais... Rio de Janeiro: ANPAD, } 2002 .\end{array}$ \\
\hline & $\begin{array}{l}\text { TOLFO, S.; PICCININI, V. A qualidade de vida no trabalho nas melhores empresas para trabalhar no Brasil: disjunções entre } \\
\text { a teoria e a prática. In: Encontro da Associação Nacional dos Programas de Pós-Graduaçãa em Administração, 26., 2002, } \\
\text { Salvador. Anais... Rio de Janeiro: ANPAD, 2002. }\end{array}$ \\
\hline 2003 & $\begin{array}{l}\text { ANDRADE, M.; CHAMON, E. Influência do sobrepeso na qualidade de vida no trabalho: estudo de caso em uma montadora } \\
\text { de automóveis. In: Encontro da Associação Nacional dos Programas de Pós-Graduação em Administração, 27., 2003, } \\
\text { Atibaia. Anais... Rio de Janeiro: ANPAD, } 2003 .\end{array}$ \\
\hline & $\begin{array}{l}\text { CARVALHO, V.; SOUZA, W. Qualidade de vida em organização de trabalho voluntário: o modelo de Hachman \& Oldham } \\
\text { aplicado à Pastoral da Criança. In: Encontro da Associação Nacional dos Programas de Pós-Graduaçãa em Administração, } \\
\text { 27., 2003, Atibaia. Anais... Rio de Janeiro: ANPAD, 2003. }\end{array}$ \\
\hline & $\begin{array}{l}\text { EL-AOUAR, W.; SOUZA, W. Com músicos, com qualidade e com vida: contribuições teórico-metodológicas aos estudos em } \\
\text { qualidade de vida no trabalho (QVT). In: Encontro da Associação Nacional dos Programas de Pós-Graduação em } \\
\text { Administração, 27., 2003, Atibaia. Anais... Rio de Janeiro: ANPAD, } 2003 .\end{array}$ \\
\hline & $\begin{array}{l}\text { GUIMARÃES, D.; MACÊDO, K. Programas de qualidade de vida no trabalho e as vivências de prazer e sofrimento dos } \\
\text { trabalhadores. In: Encontro da Associação Nacional dos Programas de Pós-Graduação em Administração, 27., 2003, Atibaia. } \\
\text { Anais... Rio de Janeiro: ANPAD, 2003. }\end{array}$ \\
\hline & $\begin{array}{l}\text { MARTINS, A. Programas de qualidade de vida, satisfação no trabalho e comprometimento organizacional. In: Encontro da } \\
\text { Associação Nacional dos Programas de Pós-Graduação em Administração, 27., 2003, Atibaia. Anais... Rio de Janeiro: } \\
\text { ANPAD, 2003. }\end{array}$ \\
\hline & $\begin{array}{l}\text { SILVA, J.; MATOS, F. Qualidade de vida no trabalho e produtividade na indústria da castanha. In: Encontro da Associação } \\
\text { Nacional dos Programas de Pós-Graduação em Administração, 27., 2003, Atibaia. Anais... Rio de Janeiro: ANPAD, } 2003 .\end{array}$ \\
\hline & $\begin{array}{l}\text { SOUZA, J.; et al. Práticas inovadoras de gestão e implementação de programas de qualidade de vida no trabalho. In: } \\
\text { Encontro da Associação Nacional dos Programas de Pós-Graduação em Administração, 27., 2003, Atibaia. Anais... Rio de } \\
\text { Janeiro: ANPAD, 2003. }\end{array}$ \\
\hline 2004 & $\begin{array}{l}\text { GUIMARÃES, D. A responsabilidade social empresarial e a precarização da qualidade de vida no trabalho de uma empresa } \\
\text { de call center. In: Encontro da Associação Nacional dos Programas de Pós-Graduação em Administração, 28., 2004, } \\
\text { Curitiba. Anais... Rio de Janeiro: ANPAD, 2004. }\end{array}$ \\
\hline & $\begin{array}{l}\text { MARQUES, A.; MORAES, L. Desenvolvimento gerencial através de cursos de longa duração: um estudo sobre a percepção } \\
\text { de eficácia dos cursos de MBA e suas relações com a qualidade de vida e estresse no trabalho. In: Encontro da Associação } \\
\text { Nacional dos Programas de Pós-Graduação em Administração, 28., 2004, Curitiba. Anais... Rio de Janeiro: ANPAD, 2004. }\end{array}$ \\
\hline & $\begin{array}{l}\text { RODRIGUES, A.; MORIN, E. Mídia e reconhecimento profissional: a análise de conteúdo como recurso para exploração de } \\
\text { práticas discursivas e impactos na qualidade de vida no trabalho de executivos. In: Encontro da Associação Nacional dos } \\
\text { Programas de Pós-Graduação em Administraçãa, 28., 2004, Curitiba. Anais... Rio de Janeiro: ANPAD, 2004. }\end{array}$ \\
\hline 2005 & $\begin{array}{l}\text { ADORNO, R.; MARQUES, A.; BORGES, R. A LDB/96 e a qualidade de vida no trabalho: com a palavra os docentes da rede } \\
\text { pública de Belo Horizonte. In: Encontro da Associação Nacional dos Programas de Pós-Graduação em Administração, 29., } \\
\text { 2005, Brasília. Anais... Rio de Janeiro: ANPAD, } 2005 .\end{array}$ \\
\hline & $\begin{array}{l}\text { BISPO, A.; et al. Empresas de economia de comunhão: em busca da qualidade de vida no trabalho? In: Encontro da } \\
\text { Associação Nacional dos Programas de Pós-Graduação em Administração, 29., 2005, Brasília. Anais... Rio de Janeiro: } \\
\text { ANPAD, 2005. }\end{array}$ \\
\hline & $\begin{array}{l}\text { DONAIRE, D.; ZACHARIAS, J.; PINTO, A. Um estudo sobre a qualidade de vida no trabalho nas agências bancárias do Vale } \\
\text { do Ribeira: uma contribuiçãa à estratégia de gestão de pessoas. In: Encontro da Associação Nacional dos Programas de } \\
\text { Pós-Graduação em Administração, 29., 2005, Brasília. Anais... Rio de Janeiro: ANPAD, 2005. }\end{array}$ \\
\hline & $\begin{array}{l}\text { DOURADO, D.; CARVALHO, C. Controle do homem no trabalho ou qualidade de vida no trabalho? In: Encontro da } \\
\text { Associação Nacional dos Programas de Pós-Graduação em Administração, 29., 2005, Brasília. Anais... Rio de Janeiro: } \\
\text { ANPAD, 2005. }\end{array}$ \\
\hline & $\begin{array}{l}\text { MOURÃO, T.; KILIMNIK, Z.; FERNANDES, E. Qualidade de vida no trabalho: um estudo de caso na Pró-reitoria de Pessoal } \\
\text { da Universidade Federal do Rio de Janeiro. In: Encontro da Associação Nacional dos Programas de Pós-Graduação em } \\
\text { Administração, 29., 2005, Brasília. Anais... Rio de Janeiro: ANPAD, 2005. }\end{array}$ \\
\hline & $\begin{array}{l}\text { SOUZA, W.; et al. Qualidade de vida no trabalho (QVT): interfaces com a qualidade em serviços no ramo da comercialização } \\
\text { de combustíveis automotivos. In: Encontro da Associação Nacional dos Programas de Pós-Graduação em Administração, } \\
\text { 29., 2005, Brasília. Anais... Rio de Janeiro: ANPAD, } 2005 .\end{array}$ \\
\hline
\end{tabular}


Para a variável base epistemológica, os artigos foram avaliados segundo seu processo discursivo preponderante, seguindo as definições apresentadas por Bruyne, Herman e Schoutheete (1977). Esses processos, segundo o autor, não se excluem mutuamente. Alguns podem ser onipresentes, outros podem não aparecer em pesquisas particulares. São eles:

- Dialética - Esforço epistemológico crítico essencial, que não explica, mas sim, prepara quadros de explicação;

- Fenomenologia - É um método de um positivismo superior, fundado sobre uma espécie de intuição, captado por uma reflexão;

- Hipotético-dedutivo - Conjunto de proposições cujos termos são rigorosamente definidos, sendo elaborado a partir de uma conceitualização da realidade percebida e observada.

Na variável base teórica foram analisados os artigos com base em quadros de referência, os quais desempenham papéis paradigmáticos implícitos, fornecendo inspirações e problemáticas a pesquisa. São eles:

- Positivismo - Recusa da apreensão imediata da realidade, ou seja, caracteriza-se pela subordinação da imaginação à observação;

- Compreensão - Busca exibir as significações internas do comportamento;

- Funcionalismo - Adota uma concepção, diferentemente da compreensão, totalizante diante dos fatos sociais;

- Estruturalismo - Possui natureza sistêmica, essencialmente construtivista e formal, afastando-se de qualquer outra significação exterior.

Como representante da base morfológica foram utilizados os seguintes quadros de análise:
- Tipologia - Contribuem para a edificação de um quadro conceitual amplamente descritivo que tem, no máximo, um poder de generalização empírica;

- Tipo ideal - Objetiva, não o recenseamento de todas as determinações de um fenômeno, mas tornar a existência desse inteligível, de um ponto de vista científico;

- Sistema - Reconhece numa problemática de pesquisa, a predominância do todo sobre as partes, abordando seu objeto de forma globalizante;

- Modelos estruturais - Polariza a pesquisa em seu aspecto morfológico sem, necessariamente, reduzir-se a ele.

Esses quadros de análise atuam, na pesquisa, como métodos de ordenação dos elementos constitutivos dos objetos científicos.

$\mathrm{Na}$ análise da variável perfil metodológico foram tomadas, como base predominante, as classificações propostas por Gil $(1991,1999)$, tendo o seguinte procedimento de classificação: procedimento de coleta de dados - qualitativo, quantitativo e qualitativo/quantitativo; níveis da pesquisa - exploratória, descritiva e explicativa, sendo acrescentado a estes mais um nível, exploratório-descritivo, colocado por Tripodi et al (1981); e procedimentos técnicos - pesquisa documental, pesquisa bibliográfica, levantamento (survey), caso e pesquisa ação.

Por fim, as variáveis 'padrão de referência' e 'demografia de autoria' foram avaliadas mediante contagem simples, usando, quando necessário, os dados fornecidos nos anais e programas de cada ENANPAD.

\section{O Que Foi Encontrado}

A apresentação dos resultados e sua discussão serão feitas conforme cada variável coletada.

\begin{tabular}{|c|c|c|c|c|c|c|c|c|}
\hline \multirow{2}{*}{$\begin{array}{ll}\text { PROCESSOS } \\
\text { DISCURSIVOS } \\
\text { ANO } \\
\end{array}$} & \multicolumn{2}{|c|}{ DIALÉTICA } & \multicolumn{2}{|c|}{ FENOMENOLOGIA } & \multicolumn{2}{|c|}{$\begin{array}{l}\text { HIPOTÉTICO- } \\
\text { DEDUTIVO }\end{array}$} & \multicolumn{2}{|c|}{ TOTAL } \\
\hline & $\mathbf{N}$ & $\%$ & $\mathbf{N}$ & $\%$ & $\mathbf{N}$ & $\%$ & $\mathbf{N}$ & $\%$ \\
\hline 2001 & - & - & - & - & 03 & 100 & 03 & 11 \\
\hline 2002 & - & - & 02 & 22 & 07 & 78 & 09 & 32 \\
\hline 2003 & - & - & 01 & 14 & 06 & 86 & 07 & 25 \\
\hline 2004 & - & - & 01 & 33 & 02 & 67 & 03 & 11 \\
\hline 2005 & 01 & 17 & - & - & 05 & 83 & 06 & 21 \\
\hline TOTAL & 01 & 04 & 04 & 14 & 23 & 82 & 28 & 100 \\
\hline
\end{tabular}

Fonte: Dados da pesquisa 
É pertinente observar uma grande concentração de artigos seguindo a lógica hipotético-dedutiva, baseada na categorização do fenômeno e no uso de técnicas quantitativas. Fator interessante a ser observado é que, trabalhos com orientação dialética ou fenomenológica, que sugerem compreensão, reflexão e interpretação, apresentaram-se em menor número, talvez, por representarem tendência sempre presente na área de Ciências Humanas, porém não dominantes na área de Administração. No quadro seguinte será possível perceber a relação entre o quadro de análise tipológico com o processo discursivo hipotético-dedutivo, assim como com o quadro de referência positivista.

Tabela 2 - Classificação dos artigos segundo os quadros de referência de Bruyne, Herman e Schoutheete (1977)

\begin{tabular}{|c|c|c|c|c|c|c|c|c|c|c|}
\hline $\begin{array}{c}\begin{array}{c}\text { QUADRO DE } \\
\text { REFERÊNCIA }\end{array} \\
\end{array}$ & \multicolumn{2}{|c|}{$\begin{array}{l}\text { POSITI- } \\
\text { VISMO }\end{array}$} & \multicolumn{2}{|c|}{$\begin{array}{c}\text { COM- } \\
\text { PREENSÃO }\end{array}$} & \multicolumn{2}{|c|}{$\begin{array}{l}\text { FUNCIO- } \\
\text { NALISTA }\end{array}$} & \multicolumn{2}{|c|}{$\begin{array}{l}\text { ESTRUTU- } \\
\text { RALISTA }\end{array}$} & \multicolumn{2}{|c|}{ TOTAL } \\
\hline & $\mathbf{N}$ & $\%$ & $\mathrm{~N}$ & $\%$ & $\mathbf{N}$ & $\%$ & $\mathrm{~N}$ & $\%$ & $\mathbf{N}$ & $\%$ \\
\hline 2001 & 02 & 67 & - & - & - & - & 01 & 33 & 03 & 11 \\
\hline 2002 & 03 & 33 & 02 & 23 & 04 & 44 & - & - & 09 & 32 \\
\hline 2003 & 05 & 72 & 01 & 14 & 01 & 14 & - & - & 07 & 25 \\
\hline 2004 & 02 & 67 & 01 & 33 & - & - & - & - & 03 & 11 \\
\hline 2005 & 05 & 83 & 01 & 17 & - & - & - & - & 06 & 21 \\
\hline TOTAL & 17 & 60 & 05 & 18 & 05 & 18 & 01 & 04 & 28 & 100 \\
\hline
\end{tabular}

Fonte: Dados da pesquisa

Pode-se verificar uma forte predominância de artigos de base positivista, isto é, que poderiam ser enquadrados como tipologias, visto que apresentaram uma estrutura conceitual analítica e uma tendência empírica, alvo da quase totalidade dos trabalhos analisados. Assim, segundo Bruyne, Herman e Schoutheete (1977), pode-se dizer que esses estudos visavam apenas determinar variáveis de uma situação que se desejava descrever.

Os trabalhos de orientação ideal-típica, ou seja, aqueles cujo quadro de referência foi a Compreensão, distribuíram-se em menor freqüência, provavelmente em virtude do próprio tema abordado pelos trabalhos. Historicamente, atribui-se a Trist e Bamforth (1951) a origem da denominação Qualidade de Vida no Trabalho QVT, sendo que até o presente momento, ainda não se conseguiu estabelecer um conceito definitivo. Sabendo que o tipo ideal é um modo de teorização, o contexto no qual se encontra o construto, tema dos trabalhos analisados, pode estar interferindo na orientação morfológica dos mesmos.

$\mathrm{Na}$ mesma proporção que no quadro Compreensão, trabalhos funcionalistas também foram encontrados, principalmente no ano de 2002, ano no qual houve um número maior de trabalhos publicados sobre o tema Qualidade de Vida no Trabalho. O quadro Funcionalista difere do Positivismo em dois critérios: o objeto de estudo em trabalhos funcionalistas é a ação social; nos trabalhos positivistas esse objeto é o fato dado, isto é, o caso. O outro critério é o objetivo do trabalho. Para os estudos funcionalistas, o objetivo é analisar as inter-relações e as causalidades entre as coisas, enquanto os positivistas têm por objetivo compreender os fatos com base em teorias.

Tabela 3 - Classificação dos artigos segundo os procedimentos de coleta de dados abordados por Gil (1991)

\begin{tabular}{c|c|c|c|c|c|c|c|c}
\hline $\begin{array}{c}\text { PROCEDIMENTO DE } \\
\text { COLETA DE DADOS }\end{array}$ & \multicolumn{2}{|c|}{$\begin{array}{c}\text { QUALI- } \\
\text { QATIVO }\end{array}$} & \multicolumn{2}{c|}{$\begin{array}{c}\text { QUANTI- } \\
\text { TATIVO }\end{array}$} & \multicolumn{2}{c|}{$\begin{array}{c}\text { QUALITATIVOI } \\
\text { QUANTITATIVO }\end{array}$} & \multicolumn{2}{c}{ TOTAL } \\
\hline ANO & $\mathbf{N}$ & $\mathbf{\%}$ & $\mathbf{N}$ & $\mathbf{\%}$ & $\mathbf{N}$ & $\%$ & $\mathbf{N}$ & $\%$ \\
\hline $\mathbf{2 0 0 1}$ & - & - & - & - & 03 & 100 & 03 & 11 \\
\hline $\mathbf{2 0 0 2}$ & 01 & 11 & 01 & 11 & 07 & 78 & 09 & 32 \\
\hline $\mathbf{2 0 0 3}$ & 01 & 14 & 02 & 29 & 04 & 57 & 07 & 25 \\
\hline $\mathbf{2 0 0 4}$ & 02 & 67 & 01 & 33 & - & - & 03 & 11 \\
\hline $\mathbf{2 0 0 5}$ & 01 & 17 & 01 & 17 & 04 & 66 & 06 & 21 \\
\hline TOTAL & $\mathbf{0 5}$ & $\mathbf{1 8}$ & $\mathbf{0 5}$ & $\mathbf{1 8}$ & $\mathbf{1 8}$ & $\mathbf{6 4}$ & $\mathbf{2 8}$ & $\mathbf{1 0 0}$ \\
\hline
\end{tabular}

Fonte: Dados da pesquisa

Percebeu-se que predominaram artigos nos quais os dados foram trabalhados quali/quantitativamente, quase sempre compostos de questionário complementado por uma entrevista semi-estruturada. Verificou-se, também, uma tendência dos trabalhos apresentados nos três últimos anos analisados a apresentarem estudos de natureza qualitativa. Entre os 28 artigos 
analisados, 23, que equivalem a $82 \%$, tomaram como base para seu estudo um modelo clássico já consagrado pela literatura, permeado ou não por outra parte qualitativa. Carvalho e Souza (2003) e El-Auoar e Souza (2003) atentam, respectivamente, para o valor do procedimento qualitativo e para barreiras e ruídos de comunicação gerados quando se direciona instrumento padronizado, em linguagem técnico-especializada, a grupos com escolaridade limitada.

\begin{tabular}{|c|c|c|c|c|c|c|c|c|c|c|}
\hline \multirow[t]{2}{*}{$\underbrace{\begin{array}{l}\text { NÍVEIS DA } \\
\text { PESQUISA }\end{array}}_{\text {ANO }}$} & \multicolumn{2}{|c|}{$\begin{array}{l}\text { EXPLORA- } \\
\text { TÓRIA }\end{array}$} & \multicolumn{2}{|c|}{$\begin{array}{l}\text { DESCRI- } \\
\text { TIVA }\end{array}$} & \multicolumn{2}{|c|}{$\begin{array}{l}\text { EXPLICA- } \\
\text { TIVA }\end{array}$} & \multicolumn{2}{|c|}{$\begin{array}{l}\text { EXPLORATÓRIO- } \\
\text { DESCRITIVA }\end{array}$} & \multicolumn{2}{|c|}{ TOTAL } \\
\hline & $\mathbf{N}$ & $\%$ & $\mathbf{N}$ & $\%$ & $\mathbf{N}$ & $\%$ & $\mathbf{N}$ & $\%$ & $\mathbf{N}$ & $\%$ \\
\hline 2001 & - & - & 02 & 67 & 01 & 33 & - & - & 03 & 11 \\
\hline 2002 & 02 & 23 & 03 & 33 & - & - & 04 & 44 & 09 & 32 \\
\hline 2003 & 02 & 29 & 02 & 29 & - & - & 03 & 42 & 07 & 25 \\
\hline 2004 & 01 & 33,33 & 01 & 33,33 & 01 & 33,33 & - & - & 03 & 11 \\
\hline 2005 & 01 & 17 & 03 & 50 & - & - & 02 & 33 & 06 & 21 \\
\hline TOTAL & 06 & 21 & 11 & 39 & 02 & 08 & 09 & 32 & 28 & 100 \\
\hline
\end{tabular}

Fonte: Dados da pesquisa

Observa-se uma distribuição quase eqüitativa entre os estudos com características descritivas e aqueles que exploravam e, ao mesmo tempo, descreviam determinada realidade, sendo as pesquisas descritivas melhor distribuídas entre os cinco anos de ENANPAD avaliados. Tem-se, portanto, que $71 \%$ dos artigos sobre QVT publicados neste novo século têm o objetivo, consoante Gil (1999), de descrever as características de determinada população ou fenômeno, ou estabelecer relação entre as variáveis, de maneira a utilizar-se de técnicas padronizadas de coleta de dados. Verificou-se também que $53 \%$ dos trabalhos avaliados apresentaram fundamentos exploratórios em sua metodologia. Esta natureza exploratória se dá, não preponderantemente em relação à teoria, há muito já estudada pela literatura da área, mas sim em relação aos campos nos quais estes estudos estão sendo realizados (telecomunicações, hotéis, Polícia etc).

Tabela 5 - Classificação dos artigos segundo o delineamento da pesquisa conforme Gil (1999)

\begin{tabular}{|c|c|c|c|c|c|c|c|c|}
\hline \multirow{2}{*}{$\begin{array}{c}\text { DELINEAMENTO DA } \\
\text { PESQUISA } \\
\text { ANO }\end{array}$} & \multicolumn{2}{|c|}{$\begin{array}{c}\text { PESQUISA } \\
\text { DOCUMENTAL }\end{array}$} & \multicolumn{2}{|c|}{$\begin{array}{l}\text { LEVANTAMENTO } \\
\text { (SURVEY) }\end{array}$} & \multicolumn{2}{|c|}{ CASO } & \multicolumn{2}{|c|}{ TOTAL } \\
\hline & $\mathbf{N}$ & $\%$ & $\mathbf{N}$ & $\%$ & $\mathbf{N}$ & $\%$ & $\mathbf{N}$ & $\%$ \\
\hline 2001 & - & - & 01 & 33 & 02 & 67 & 03 & 11 \\
\hline 2002 & 01 & 12 & 04 & 44 & 04 & 44 & 09 & 32 \\
\hline 2003 & - & - & 05 & 71 & 02 & 29 & 07 & 25 \\
\hline 2004 & 01 & 33,33 & 01 & 33,33 & 01 & 33,33 & 03 & 11 \\
\hline 2005 & 01 & 17 & 02 & 33 & 03 & 50 & 06 & 21 \\
\hline TOTAL & 03 & 11 & 13 & 46 & 12 & 43 & 28 & 100 \\
\hline
\end{tabular}

Fonte: Dados da pesquisa

Quanto ao delineamento da pesquisa observouse que algumas formas de classificação adotadas por Gil (1999) aparecem de maneira secundária nos trabalhos analisados: pesquisa bibliográfica e observação participante. Enquanto outras classificações sequer foram observadas no estudo como, por exemplo, a pesquisa experimental e expost-facto, ambas com características de laboratório, além de outra classificação adotada por Gil (1991), como a pesquisa ação. Ficou evidente, portanto, o predomínio de artigos que se apóiam sobre a técnica survey, ou seja, um levantamento de dados feito pela interrogação direta das pessoas cujo comportamento se deseja conhecer, para em seguida, mediante análise quantitativa, obter as conclusões correspondentes.

Logo em seguida ficaram os artigos que trabalharam mediante estudo de caso, os quais possuem base empírica de investigação. Esses tipos de artigo mostraram-se em declínio, de 2002 a 2004, talvez em virtude de alguns preconceitos adquiridos pelos pesquisadores em relação a este tipo de estudo, tais como: falta de rigor metodológico, dificuldade de generalização e um grande espaço de tempo a ser destinado a pesquisa. Esses preconceitos talvez tivessem sido incorporados pela Associação Nacional de Programas de Pós-graduação em Administração (ANPAD), o que explicaria a redução do número de 
estudos de caso no Encontro Científico da mesma. Em 2005, no entanto, o número de estudos dessa natureza acerca do tema qualidade de vida no trabalho voltou a aumentar.

\begin{tabular}{|c|c|c|c|c|c|c|c|c|}
\hline \multirow{2}{*}{ ANO } & \multicolumn{2}{|c|}{ ARTIGOS } & \multicolumn{2}{|c|}{ LIVROS } & \multicolumn{2}{|c|}{ OUTROS } & \multicolumn{2}{|c|}{ TOTAL } \\
\hline & $\mathbf{N}$ & $\%$ & $\mathbf{N}$ & $\%$ & $\mathbf{N}$ & $\%$ & $\mathbf{N}$ & $\%$ \\
\hline 2001 & 24 & 44 & 15 & 27 & 16 & 29 & 55 & 100 \\
\hline 2002 & 70 & 29 & 119 & 49 & 54 & 22 & 243 & 100 \\
\hline 2003 & 57 & 33 & 81 & 48 & 32 & 19 & 170 & 100 \\
\hline 2004 & 22 & 27 & 42 & 52 & 17 & 21 & 81 & 100 \\
\hline 2005 & 59 & 39 & 65 & 43 & 26 & 18 & 150 & 100 \\
\hline TOTAL & 232 & 33 & 322 & 46 & 145 & 21 & 699 & 100 \\
\hline
\end{tabular}

Fonte: Dados da pesquisa

Quanto às referências, ao contrário do que foi verificado por Caldas, Tonelli e Lacombe (2002) analisando a produção científica em recursos humanos nos ENANPADs da década de 90, verificou-se uma tendência de maior utilização de livros durante os quatro últimos anos analisados pelo presente estudo e uma correspondente diminuição na utilização de artigos.

Importante questionar até que ponto essa diminuição pode afetar a atualidade do que está sendo pesquisado. Não se pretende, com esse questionamento, diminuir a grande importância que as referências bibliográficas têm para qualquer estudo. Não se pode, contudo, negar o fato de que o tempo estimado para uma publicação de livro é bem maior que de um artigo, o que acaba tornando o conteúdo deste último mais recente. Outros tipos de referências incluem relatórios de pesquisa, documentos, artigos publicados em jornais e revistas não científicas e, principalmente, dissertações e teses.

Verificou-se, ainda, a produção por instituição e os autores que mais trabalhos apresentaram no período abordado pelo presente estudo. Em relação à produção por instituição, notou-se uma distribuição relativamente igualitária, despontando apenas a UFMG (Universidade Federal de Minas Gerais), com sete artigos, a UFRN (Universidade Federal do Rio Grande do Norte) e a UFRGS (Universidade Federal do Rio Grande do Sul) com três, cada uma; a UCG (Universidade Católica de Goiás) e a UFPE (Universidade Federal de Pernambuco), com dois artigos cada uma. As demais mantêm o número absoluto de trabalhos relativamente constante.

Analisando esses dados de acordo com o processo discursivo utilizado nos artigos, foi possível verificar que todos os trabalhos originados da UFMG foram desenvolvidos à luz da lógica hipotético-dedutiva. Enquanto isso, estudos realizados por pesquisadores da UFRN e UFRGS mostraram-se divididos entre o método fenomenológico e o hipotético-dedutivo. A UCG também foi caracterizada pela realização de pesquisas de cunho hipotético-dedutivo, enquanto a UFPE apresentou estudos tanto de natureza dialética, quanto seguidores da lógica hipotéticodedutiva. Quanto aos autores que mais trabalhos apresentaram no período, verifica-se que a distribuição acompanha a das instituições a que estão filiados.

\section{Onde se Pôde Chegar}

Dentro do cenário de pesquisas epistemológicas, o objeto desta investigação contemplou a avaliação dos trabalhos publicados no ENANPAD acerca do tema Qualidade de Vida no Trabalho nos anos de 2001 a 2005, com ênfase na análise das bases epistemológica, teórica e morfológica, assim como no levantamento de um perfil metodológico.

Foi visto anteriormente que publicações sobre QVT nos EnANPADs estudados apresentavam problemas e desafios semelhantes aos colocados por estudos já realizados na área de Recursos Humanos - Roesch, Antunes e Silva (1997); Caldas, Tonelli e Lacombe (2002); Tonelli et al (2004). O presente estudo revela a imagem de uma produção com volume considerável de artigos nos últimos anos, principalmente na área de comportamento organizacional, na qual, segundo Tonelli et al (2004), o tema registra uma tendência de aumento da representatividade no total dos trabalhos apresentados.

Foi possível observar, dentro desse contexto, que a maior parte dos trabalhos que vem sendo desenvolvidos acerca do tema Qualidade de Vida no Trabalho apresenta um caráter empirista analítico, ou seja, corresponde de fato a um estágio pouco avançado do desenvolvimento de uma disciplina científica, contribuindo para a edificação de um quadro conceitual amplamente descritivo 
que tem, no máximo, um poder de generalização empírica.

Todavia, alguma evolução tem sido percebida quando se observa a crítica colocada pelos autores anteriormente citados. Esses autores questionam a metodologia empregada nos trabalhos da área de Recursos Humanos, tendo em vista apresentarem predominância de pesquisa qualitativa sem maior pretensão indutiva; o emprego de estudos de caso está concentrado em um conjunto extremamente limitado de autores e programas. Nos estudos sobre artigos que versam sobre QVT o que foi visto foi uma preferência por estudos mistos (qualitativo/quantitativo), tendo em vista a tendência a considerar que estudos apenas quantitativos acabam gerando uma limitação para a investigação (EL-AUOAR e SOUZA, 2003).

Quanto ao emprego do estudo de caso, o presente trabalho corrobora com a opinião de Yin (1981). Este autor argumenta que vieses, como falta de rigor metodológico, colocados pelos que criticam o estudo de caso, não são prerrogativas desses, podendo ocorrer em outras modalidades de pesquisa. Logo, o que se propõe ao pesquisador é que redobre seus cuidados no planejamento da pesquisa e em sua coleta de dados.

No que se refere à dificuldade de generalização, também colocado como crítica ao estudo de caso, é posto que os propósitos desse tipo de estudo não são proporcionar o conhecimento preciso das características de uma população, a partir de procedimentos estatísticos, mas sim expandir ou generalizar proposições teóricas. O autor finaliza esclarecendo que um bom estudo de caso é tarefa difícil de realizar, exemplificando que pesquisadores inexperientes, entusiasmados pela flexibilidade metodológica da técnica, ao final de sua pesquisa têm apenas uma grande quantidade de dados que não conseguem analisar nem interpretar.

É possível esclarecer, também, dentro de uma perspectiva de evolução científica, que dentre os artigos analisados, existiu considerável diversificação de autores e universidades. Observase ainda uma tendência à publicação em conjunto, uma vez que apenas três trabalhos foram publicados por um único autor.

Não foi alvo da presente investigação estudar em quais setores vêm se desenvolvendo tais pesquisas, assim como não foram feitas análises bibliométricas, nem se procurou avaliar o conteúdo teórico dos artigos, o que fica como questões a serem respondidas por futuras pesquisas.

Referências
ARKADER, R. A pesquisa científica em gerência de operações no Brasil. Revista de Administração de Empresas, v. 43, n. 1, p. 70-80, jan./mar, 2003.

BASTOS, A. V. B. A produção científica em recursos humanos - temas e metodologias dos trabalhos apresentados nos principais periódicos nacionais entre 1983 e 1988. In: ENCONTRO DA ASSOCIAÇÃO NACIONAL DOS PROGRAMAS DE PÓS-GRADUAÇÃO EM ADMINISTRAÇÃO, 13., 1989, São Paulo. Anais... Rio de Janeiro: ANPAD, 1989.

BERTERO, C. O.; CALDAS, M. e WOOD Jr, T. Produção científica em administração de empresas: provocações, insinuações e contribuições para um debate local. Revista de Administração

Contemporânea, v. 3, n. 1, p. 147-178, jan./abr., 1999.

BIGNETTI, L. P.; PAIVA, E. L. Ora (direis) ouvir estrelas! Estudo das citações de autores de estratégia na produção acadêmica brasileira. Revista de Administração Contemporânea, v. 6, n. 1, p. 105-125, jan./abr, 2002.

BRUYNE, P. de; HERMAN, J.; SCHOUTHEETE, M. de. Dinâmica de pesquisa em ciências sociais: os pólos da prática epistemológica. Rio de Janeiro: Francisco Alves, 1977.

CALDAS, M. P.; ONKEN, M.; TINOCO, T. Is business ethics research ethnocentric? an analysis of the extent of internationalization of the research published in top Business Ethics Journals from 1991-2002. In: ENCONTRO DA ASSOCIAÇÃO NACIONAL DOS PROGRAMAS DE PÓSGRADUAÇÃO EM ADMINISTRAÇÃO, 29., 2005, Brasília. Anais... Rio de Janeiro: ANPAD, 2005.

CALDAS, M. P.; TINOCO, T.; CHU, R. A. Análise bibliométrica dos artigos de RH publicados no ENANPAD na década de 1990 - Um mapeamento a partir das citações dos heróis, endogenias e jactâncias que fizeram a história recente da produção científica da área. In: ENCONTRO DA ASSOCIAÇÃO NACIONAL DOS PROGRAMAS DE PÓS-GRADUAÇÃO EM ADMINISTRAÇÃO, 27., 2003, São Paulo. Anais... Rio de Janeiro: ANPAD, 2003.

CALDAS, M. P.; TONELLI, M. J.; LACOMBE, B. M. B. Espelho, espelho meu: meta-estudo da produção científica em recursos humanos nos ENANPADs da década de 90. In: ENCONTRO DA ASSOCIAÇÃO NACIONAL DOS PROGRAMAS DE PÓSGRADUAÇÃO EM ADMINISTRAÇÃO, 26., 2002, Salvador. Anais... Rio de Janeiro: ANPAD, 2002.

CAMARGOS, M. A.; COUTINHO Ed. S.; AMARAL, H. F. O perfil da área de finanças do ENANPAD: 
um levantamento da produção científica e de suas tendências entre 2000-2004. In: ENCONTRO DA ASSOCIAÇÃO NACIONAL DOS PROGRAMAS DE PÓS-GRADUAÇÃO EM ADMINISTRAÇÃO, 29., 2005, Brasília. Anais... Rio de Janeiro: ANPAD, 2005.

CARVALHO, V.; SOUZA, W. J. de. Qualidade de vida em organização de trabalho voluntário: o modelo de Hachman \& Oldham aplicado à Pastoral da Criança. In: ENCONTRO DA ASSOCIAÇÃO NACIONAL DOS PROGRAMAS DE PÓSGRADUAÇÃO EM ADMINISTRAÇÃO, 27., 2003, São Paulo. Anais... Rio de Janeiro: ANPAD, 2003.

COSTA, L. S.V. Por que, com quem, como, quando, até quando? estratégias colaborativas: a quantas anda essa "colcha de retalhos". In: ENCONTRO DA ASSOCIAÇÃO NACIONAL DOS PROGRAMAS DE PÓS-GRADUAÇÃO EM ADMINISTRAÇÃO, 29., 2005, Brasília. Anais... Rio de Janeiro: ANPAD, 2005.

EL-AOUAR, W. A.; SOUZA, W. J. de. Com músicos, com qualidade e com vida: contribuições teórico-metodológicas ao estudo da qualidade de vida no trabalho (QVT). In: ENCONTRO DA ASSOCIAÇÃO NACIONAL DOS PROGRAMAS DE PÓS-GRADUAÇÃO EM ADMINISTRAÇÃO, 27. 2003, São Paulo. Anais... Rio de Janeiro: ANPAD, 2003.

FROEMMING, L. M. S et al. Análise da qualidade dos artigos científicos da área de marketing no Brasil: as pesquisas survey na década de 90.

Revista de Administração Contemporânea. v. 4, n. 3, Set./Dez. 2000, p. 201-219.

FROEMMING, L. M. S et al. Inventário de artigos científicos na área de marketing no Brasil. Revista de Administração Contemporânea. v. 4, n. 2, Maio/Ago. 2000b, p. 159-173.

GIL, A. C. Como elaborar projetos de pesquisa. São Paulo: Atlas, 1991.

GIL, A. C. Métodos e técnicas de pesquisa social. São Paulo: Atlas, 1999.

GONÇALVES, N. G., GONÇALVES, S. A., AUGUSTO, P. O. M. Sobre a relação Estado e educação na produção acadêmica brasileira (19712000): temas, críticas e expectativas. In: ENCONTRO DA ASSOCIAÇÃO NACIONAL DOS PROGRAMAS DE PÓS-GRADUAÇÃO EM ADMINISTRAÇÃO, 27., 2003, São Paulo. Anais... Rio de Janeiro: ANPAD, 2003.

GOSLING, M.; GONÇALVES, C. A. Idéias metodológicas dos autores de estratégia dos enanpads: uma meta-análise. REAd, ed. 41, v. 10, n. 5 , set/out. 2004.
HEMAIS, B.; VERGARA, S. O jeito brasileiro de publicar em estudos organizacionais. Revista Organizações e Sociedade, v. 8, n. 20, jan./mar., 2001.

HOPPEN, N.; et al. Sistemas de informação no Brasil: uma análise dos artigos científicos dos anos 90. In: ENCONTRO DA ASSOCIAÇÃO NACIONAL DOS PROGRAMAS DE PÓS-GRADUAÇÃO EM ADMINISTRAÇÃO, 22., 1998, Foz do Iguaçu. Anais... Rio de Janeiro: ANPAD, 1998.

KEINERT, T. M. O que é administração pública no Brasil? In: In: ENCONTRO DA ASSOCIAÇÃO NACIONAL DOS PROGRAMAS DE PÓSGRADUAÇÃO EM ADMINISTRAÇÃO, 24., 1998, Florianópolis. Anais... Rio de Janeiro: ANPAD, 2000.

KEINERT, T. M. A produção em administração pública na ANPAD (1976-1992): uma análise preliminar. In: ENCONTRO DA ASSOCIAÇÃO NACIONAL DOS PROGRAMAS DE PÓSGRADUAÇÃO EM ADMINISTRAÇÃO, 18., 1994, Curitiba. Anais... Rio de Janeiro: ANPAD, 1994.

KIRSHBAUM, C.; PORTO, E. C.; FERREIRA, F. C. M. Neo-institucionalismo na produção acadêmica em administração. RAE-eletrônica, v. 3, n. 1, Art. 12, jan./jun. 2004.

LEAL, R. P. C.; OLIVEIRA, J.; SOLURI, A. F. Perfil da pesquisa em finanças no Brasil. Revista de Administração de Empresas, v. 43, n. 1. Jan./mar., 2003, p. 91-104.

LUDMER, G. et al. Conhecimento emancipatório em sistemas de informação no Brasil: uma avaliação da produção acadêmica. In: ENCONTRO DA ASSOCIAÇÃO NACIONAL DOS PROGRAMAS DE PÓS-GRADUAÇÃO EM ADMINISTRAÇÃO, 26., 2002, Salvador. Anais... Rio de Janeiro: ANPAD, 2002.

LUNARDI, G. L.; RIOS, L. R.; MAÇADA, A. C. F. Pesquisa em sistemas de informação: uma análise a partir dos artigos publicados no Enanpad e nas principais revistas nacionais de administração. In: ENCONTRO DA ASSOCIAÇÃO NACIONAL DOS PROGRAMAS DE PÓS-GRADUAÇÃO EM ADMINISTRAÇÃO, 29., 2005, Brasília. Anais... Rio de Janeiro: ANPAD, 2005.

MACHADO, C.; CUNHA, V. C.; AMBONI, N. Administração pública: uma revisão dos artigos apresentados à ANPAD no período de 1983 a 1986. In: ENCONTRO DA ASSOCIAÇÃO NACIONAL DOS PROGRAMAS DE PÓSGRADUAÇÃO EM ADMINISTRAÇÃO, 12., 1988, Natal. Anais... Rio de Janeiro: ANPAD, 1988. 
MACHADO, C.; CUNHA, V. C.; AMBONI, N. A produção acadêmica de administração pública: período 1983-1988. In: ENCONTRO DA ASSOCIAÇÃO NACIONAL DOS PROGRAMAS DE PÓS-GRADUAÇÃO EM ADMINISTRAÇÃO, 13., 1989, São Paulo. Anais... Rio de Janeiro: ANPAD, 1989.

MACHADO, C.; CUNHA, V. C.; AMBONI, N. Organizações: o estado da arte da produção acadêmica no Brasil. In: ENCONTRO DA ASSOCIAÇÃO NACIONAL DOS PROGRAMAS DE PÓS-GRADUAÇÃO EM ADMINISTRAÇÃO, 14., 1990, Florianópolis. Anais... Rio de Janeiro: ANPAD, 1990.

MARTINS, G. Epistemologia da pesquisa em administração. In: ENCONTRO DA ASSOCIAÇÃO NACIONAL DOS PROGRAMAS DE PÓSGRADUAÇÃO EM ADMINISTRAÇÃO, 20., 1996, Rio de Janeiro. Anais... Rio de Janeiro: ANPAD, 1996.

MARTINS, G. Metodologia da pesquisa em administração. In: ENCONTRO DA ASSOCIAÇÃO NACIONAL DOS PROGRAMAS DE PÓSGRADUAÇÃO EM ADMINISTRAÇÃO, 17., 1993, Salvador. Anais... Rio de Janeiro: ANPAD, 1993.

MATTOS, P. L. A estruturação de dissertações e teses em administração: caracterização teórica e sugestões práticas. Revista de Administração Contemporânea. v. 6, n. 3, Set./Dez. 2002, p. 175198.

PAIVA, Jr, F. G. CORDEIRO, A. T.

Empreendedorismo e o espírito empreendedor: uma análise da evolução dos estudos na produção acadêmica brasileira. In: ENCONTRO DA ASSOCIAÇÃO NACIONAL DOS PROGRAMAS DE PÓS-GRADUAÇÃO EM ADMINISTRAÇÃO, 26., 2002, Salvador. Anais... Rio de Janeiro: ANPAD, 2002.

PASSADOR, C. S.; CANOPF, L.; PASSADOR, J. L. Apontamentos sobre a responsabilidade social no ENANPAD: a construção de um conceito? In: ENCONTRO DA ASSOCIAÇÃO NACIONAL DOS PROGRAMAS DE PÓS-GRADUAÇÃO EM ADMINISTRAÇÃO, 29., 2005, Brasília. Anais... Rio de Janeiro: ANPAD, 2005.

PEGINO, P. M. F. As bases filosóficas das publicações na área de estratégia das organizações nos encontros nacionais da Anpad. In: ENCONTRO DA ASSOCIAÇÃO NACIONAL DOS PROGRAMAS DE PÓS-GRADUAÇÃO EM ADMINISTRAÇÃO, 29., 2005, Brasília. Anais... Rio de Janeiro: ANPAD, 2005.
PERIN, M. G., SAMPAIO, C. H., FROEMMING, L. M. S., LUCE, F. B. A pesquisa survey em artigos de marketing nos Enanpads da década de 90. In: ENCONTRO DA ASSOCIAÇÃO NACIONAL DOS PROGRAMAS DE PÓS-GRADUAÇÃO EM ADMINISTRAÇÃO, 24., 2000, Florianópolis. Anais... Rio de Janeiro: ANPAD, 2000.

PRADO JÚNIOR, S. T.; LOEBEL, E. Uma análise dos estudos sobre a dimensão política em estratégia de negócios. In: ENCONTRO DA ASSOCIAÇÃO NACIONAL DOS PROGRAMAS DE PÓS-GRADUAÇÃO EM ADMINISTRAÇÃO, 29., 2005, Brasília. Anais... Rio de Janeiro: ANPAD, 2005.

PROENÇA, E. R.; LOPES, F. T. P.; MEUCCI, A. A construção do conhecimento em marketing: considerações sobre o discurso acadêmico e a produção científica do I encontro de marketing da ANPAD. In: ENCONTRO DA ASSOCIAÇÃO NACIONAL DOS PROGRAMAS DE PÓSGRADUAÇÃO EM ADMINISTRAÇÃO, 29., 2005, Brasília. Anais... Rio de Janeiro: ANPAD, 2005.

RAMOS, A. S. M.; SILVA, S. M. C.; SALES, C. A. C. Produção científica na área de internet na administração: uma revisão de literatura e classificação de artigos nos anais do Enanpad de 1997 a 2003. In: Seminário de Pesquisa do Centro de Ciências Sociais Aplicadas, 10., 2004, Natal. Anais... Natal: CCSA, 2004.

RODRIGUES FILHO, J. Um estudo da produção acadêmica em administração estratégica no Brasil na terminologia de Habermas. RAE-eletrônica, v. 3, n. 2, Art. 21, jul./dez. 2004

ROESCH, S., ANTUNES, E. e SILVA, L. V. Tendências da pesquisa em recursos humanos e organizações: uma análise das dissertações de mestrado. In: ENCONTRO DA ASSOCIAÇÃO NACIONAL DOS PROGRAMAS DE PÓSGRADUAÇÃO EM ADMINISTRAÇÃO, 21., 1997, Rio de Janeiro. Anais... Rio de Janeiro: ANPAD, 1997.

SIQUEIRA, M. M. O tema recursos humanos nas reuniões da ANPAD: trajetória e perspectivas. In: ENCONTRO DA ASSOCIAÇÃO NACIONAL DOS PROGRAMAS DE PÓS-GRADUAÇÃO EM ADMINISTRAÇÃO, 12., 1988, Natal. Anais... Rio de Janeiro: ANPAD, 1988.

THEÓPHILO, C. R.; IUDÍCIBUS S. Uma análise crítico-epistemológica da produção científica em contabilidade no Brasil. In: ENCONTRO DA ASSOCIAÇÃO NACIONAL DOS PROGRAMAS DE PÓS-GRADUAÇÃO EM ADMINISTRAÇÃO, 29., 
2005, Brasília. Anais... Rio de Janeiro: ANPAD, 2005.

TRIPODI, T. et al. Análise da pesquisa social. Rio de Janeiro: Francisco Alves, 1981.

TRIST, E. L.; BAMFORTH, K. W. Some social and psychological consequences of the longwall method of coal-getting. Human Relations. v. 4, n. 1, p. 338, 1951.

TONELLI, M. J. et al. Produção acadêmica em recursos humanos no Brasil: 1991-2000. Revista de Administração de Empresas, vol. 43, n.1, p. 105-122, 2003.

TONELLI, M. J. et al. O mapa da partilha: análise das áreas de comportamento organizacional e gestão de pessoas antes e depois da cisão da área de "recursos humanos" no ENANPAD, 1991-2003. In: ENCONTRO DA ASSOCIAÇÃO NACIONAL DOS PROGRAMAS DE PÓS-GRADUAÇÃO EM ADMINISTRAÇÃO, 28., 2004, Curitiba. Anais... Rio de Janeiro: ANPAD, 2004.

VIEIRA, F. G. D. Por quem os sinos dobram? Uma análise da publicação científica na área de marketing do Enanpad. In: ENCONTRO DA ASSOCIAÇÃO NACIONAL DOS PROGRAMAS DE PÓS-GRADUAÇÃO EM ADMINISTRAÇÃO, 22., 1998, Foz do Iguaçu. Anais... Rio de Janeiro: ANPAD, 1998.

VIEIRA, F. G. D. Ações empresariais e prioridades de pesquisa em marketing: tendências no Brasil e no mundo segundo a percepção dos acadêmicos brasileiros. In: ENCONTRO DA ASSOCIAÇÃO NACIONAL DOS PROGRAMAS DE PÓSGRADUAÇÃO EM ADMINISTRAÇÃO, 23., 1999, Foz do Iguaçu. Anais... Rio de Janeiro: ANPAD, 1999.

VIEIRA, F. G. D. Panorama acadêmico-científico e temáticas de estudos de marketing no Brasil. In: ENCONTRO DA ASSOCIAÇÃO NACIONAL DOS PROGRAMAS DE PÓS-GRADUAÇÃO EM ADMINISTRAÇÃO, 24., 2000, Florianópolis. Anais... Rio de Janeiro: ANPAD, 2000.

VIEIRA, F. G. D. Narciso sem espelho: a publicação brasileira de marketing. Revista de Administração de Empresas. 43 (1) Jan/Mar. 2003, p. 81-90.

YIN, R. K. The case study crisis: some answers. Administrative Science Quarterly, Cornell University, 26, Mar. 1981. 